\title{
The TAM family receptor tyrosine kinase TYRO3 is a negative regulator of type 2 immunity
}

\author{
Pamela Y. Chan ${ }^{1}$, Eugenio A. Carrera Silva ${ }^{1,2}$, Dimitri De Kouchkovsky ${ }^{1}$, Leonel D. \\ Joannas ${ }^{1}$, Liming $\mathrm{Hao}^{3}$, Donglei $\mathrm{Hu}^{4}$, Scott Huntsman ${ }^{4}$, Celeste Eng ${ }^{4}$, Paula Licona- \\ Limón ${ }^{1,{ }^{*}}$, Jason S. Weinstein ${ }^{1}$, De'Broski R. Herbert ${ }^{5}$, Joseph E. Craft ${ }^{1,6}$, Richard A. \\ Flavell $^{1,7}$, Silvia Repetto 8,9 , Jorge Correale ${ }^{10}$, Esteban G. Burchard ${ }^{4,11}$, Dara G. Torgerson ${ }^{4}$, \\ Sourav Ghosh ${ }^{12}$, and Carla V. Rothlin ${ }^{1, \dagger}$ \\ ${ }^{1}$ Department of Immunobiology, School of Medicine, Yale University, New Haven, CT 06520, USA \\ ${ }^{2}$ Laboratorio de Trombosis Experimental, Instituto de Medicina Experimental, Academia Nacional \\ de Medicina-CONICET, Buenos Aires, 1425, Argentina \\ ${ }^{3}$ Department of Pathology, School of Medicine, Yale University, New Haven, CT 06520, USA \\ ${ }^{4}$ Department of Medicine, University of California San Francisco, CA 94158, USA \\ ${ }^{5}$ Department of Experimental Medicine, University of California San Francisco, CA 94158, USA \\ ${ }^{6}$ Department of Internal Medicine (Rheumatology), School of Medicine, Yale University, New \\ Haven, CT 06520, USA \\ ${ }^{7}$ Howard Hughes Medical Institute, School of Medicine, Yale University, New Haven, CT 06520, \\ USA \\ 8Instituto de Investigaciones en Microbiología y Parasitología Médica, University of Buenos Aires- \\ CONICET, Buenos Aires, 1121, Argentina \\ ${ }^{9}$ Hospital de Clinicas Jose de San Martin, University of Buenos Aires, 1120, Argentina \\ ${ }^{10}$ Center for Research on Neuroimmunological Diseases, Raúl Carrea Institute for Neurological \\ Research (FLENI), Buenos Aires 1428, Argentina \\ ${ }^{11}$ Department of Bioengineering, School of Pharmacy, University of California San Francisco, CA \\ 94158, USA \\ ${ }^{12}$ Department of Neurology, School of Medicine, Yale University, New Haven, CT 06520, USA
}

\section{Abstract}

\footnotetext{
${ }_{*}^{\dagger}$ Corresponding author. carla.rothlin@yale.edu (C.V.R.).

* Present address: Departamento de Biología Celular y del Desarrollo, Instituto de Fisiología Celular, National Autonomous University of Mexico, D.F. México.

SUPPLEMENTARY MATERIALS

www.sciencemag.org/content/352/6281/99/suppl/DC1

Materials and Methods

Figs. S1 to S10

Table S1

References (21-32)
} 
Host responses against metazoan parasites or an array of environmental substances elicit type 2 immunity. Despite its protective function, type 2 immunity also drives allergic diseases. The mechanisms that regulate the magnitude of the type 2 response remain largely unknown. Here, we show that genetic ablation of a receptor tyrosine kinase encoded by Tyro 3 in mice or the functional neutralization of its ortholog in human dendritic cells resulted in enhanced type 2 immunity. Furthermore, the TYRO3 agonist PROS1 was induced in T cells by the quintessential type 2 cytokine, interleukin-4. T cell-specific Pros 1 knockouts phenocopied the loss of Tyro3. Thus, a PROS1-mediated feedback from adaptive immunity engages a rheostat, TYRO3, on innate immune cells to limit the intensity of type 2 responses.

The type 2 immune response is the major protective mechanism against metazoan parasites and is simultaneously characteristic of allergy and allergic diseases (1). The mechanism that regulates the intensity of this response is not well understood. Because genetic predisposition is an important risk factor for allergic diseases, we performed a meta-analysis of three independent genome-wide association studies (GWASs) of Latinos and African Americans with asthma. Multiple intronic variants in TYRO3 were associated with asthma (fig. S1A and table S1). The most significant association was at a single-nucleotide polymorphism (SNP) located within several putative transcription factor-binding sites in TYRO3 (fig. S1, A and B). TYRO3 is a member of the TAM (TYRO3, AXL, and MERTK) receptor tyrosine kinase (RTK) subgroup. AXL and MERTK have recently been identified as pleiotropic negative regulators of the immune response (1-4), but our meta-analysis did not reveal an association with asthma (fig. S1, C and D). The molecular functions of TYRO3 in immune regulation remain largely uncharacterized. Because TYRO3 shows substantial sequence identity with $A X L$ and MERTK, we hypothesized that TYRO3 may limit the magnitude of the immune response in the context of type 2 immunity.

To test this hypothesis, we first used a house dust mite (HDM) model of allergic airway inflammation. HDM-sensitized wild-type (WT) mice developed classical signs of allergic asthma, including influx of eosinophils and lymphocytes in the bronchoalveolar lavage (BAL) fluid and lung (Fig. 1A and fig. S2, A to C), as well as expansion of CD4 ${ }^{+} \mathrm{T}$ cells and effector memory cells in the mediastinal lymph nodes (medLNs) (fig. S2D). We also challenged mice lacking either TYRO3 alone or both AXL and MERTK with HDM. Remarkably, HDM-sensitized Tyro $3^{--}$mice displayed a more robust type 2 response, as reflected by a further increase in leukocytes and eosinophils in BAL fluid and lung (Fig. 1A and fig. S2C). This was accompanied by increased total and effector memory $\mathrm{CD}^{+} \mathrm{T}$ cells in the medLNs (fig. S2D); higher percentages of $\mathrm{CD} 4^{+} \mathrm{T}$ cells producing type 2 cytokinessuch as interleukin 4 (IL-4), IL-5, and IL-13-but not the type I cytokine interferon- $\gamma$ (IFN$\gamma)$ (Fig. 1B) and higher serum immunoglobulin E (IgE) (Fig. 1C). These exacerbated cellular and humoral type 2 responses correlated with increased lung histopathological score (Fig. 1D). A role of AXL has been described in respiratory infections $(5,6)$. However, the ablation of this receptor in conjunction with MERTK $\left(\mathrm{AxI}^{-/-} \mathrm{Mertk}^{-/-}\right)$did not result in increased susceptibility of mice to HDM (Fig. 1A and fig. S2).

To further evaluate if Tyro 3 was an essential component of the type 2 immune response, we infected mice with the helminth Nippostrongylus brasiliensis. Infected Tyro $3^{-/-}$mice had 
fewer BAL red blood cells (RBCs), which indicated reduced damage (Fig. 2A). We also observed increased BAL eosinophils and enhanced alternative activation of lung interstitial macrophages (Fig. 2B and fig. S3, A and B). Tyro3 ${ }^{-/-}$mice exhibited an accelerated and enhanced $\mathrm{T}$ cell response in the medLNs (fig. S3C), increased IL- $4^{+}$and IL- $5^{+} \mathrm{CD} 4^{+} \mathrm{T}$ cells in the medLNs (Fig. 2C), larger amounts of type 2 cytokines from mesenteric LNs (mLNs) (fig. S3D), and enhanced L3- and L5-specific IgG1 antibodies and total IgE humoral response (Fig. 2D). By days 2, 3, and 7, Tyro ${ }^{-/-}$mice had fewer worms in the lungs and small intestines, which indicates accelerated clearance of the parasite (Fig. 2E). No differences in worm burden were noted in the skin of WT and Tyro $3^{-/}$mice (fig. S3E). Furthermore, ovalbumin with papain (OVA-papain) immunization, an alternative type 2 immunity model, produced an accelerated and enhanced response in Tyro $3^{-1}$ mice (fig. S4). We did not, however, detect increased $\mathrm{T}$ helper $1\left(\mathrm{~T}_{\mathrm{H}} 1\right)$ responses in Tyro $3^{-/}$mice (fig. S5). Taken together, these results demonstrate that TYRO3 is an essential, selective inhibitor of type 2 immunity.

Next, we investigated the cellular basis of TYRO3-mediated negative regulation of type 2 immunity. $\mathrm{T}_{\mathrm{H}} 2$ differentiation is regulated by dendritic cells (DCs), the professional antigenpresenting cells (APCs) (7-10). A specific population of DCs that are CD11c-positive and programmed cell death protein 2 (PDL2)-positive $\left[\mathrm{CD} 11 \mathrm{c}^{+} \mathrm{PDL} 2^{+}\right]$has been described to induce type 2 immune responses (11-14). Bone marrow (BM)-derived CD11 ${ }^{+}$PDL2 ${ }^{+}$DCs expressed TYRO3. Moreover, TYRO3 expression was not restricted to PDL2 ${ }^{+}$DCs as PDL2- DCs also expressed this RTK (Fig. 3A). Likewise, TYRO3 was detected in lung DCs of mice sensitized with HDM extract or infected with $N$. brasiliensis, albeit not in alveolar macrophages, eosinophils, or neutrophils (fig. S6).

To test if TYRO3 functions specifically in DCs to limit the magnitude of type 2 immunity, we used an adoptive transfer model (fig. S7A). In accordance with an inhibitory role of TYRO3 in DCs, mice receiving OVA-loaded Tyro $^{-1-}{ }^{-}$PDL $2^{+}$DCs exhibited increased LN cellularity and type 2 cytokine production (Fig. 3, B and C). Note that similar percentages of WT and Tyro $^{-1-}{ }^{-} \mathrm{PDL}_{2}{ }^{+}$DCs were detected in the draining LNs, which indicated that WT and Tyro $^{-/-}$DCs migrated and survived to equal degree (fig. S7B). The type 2 immune response in mice receiving OVA-loaded WT PDL2- DCs was negligible. In contrast loss of TYRO3 in PDL2- DCs resulted in an enhanced type 2 immune response yet lower in magnitude relative to mice receiving Tyro ${ }^{-/-}$PDL2 ${ }^{+}$DCs (Fig. 3, B and C). When OVAloaded WT or Tyro $3^{-/-}$DCs were transferred during priming, only Tyro $3^{-/} \mathrm{PDL}^{+}$DCs led to an enhanced response in recipient mice (fig. S8).

Comparison of the most differentially transcribed genes between DCs that can induce type 2 immunity and those that cannot revealed that a subset of chemokines-CCL22, CCL17, and CCL5-are highly expressed in PDL2+ DCs (11). Some of these chemokines actively promote type 2 immunity, as neutralization of their function results in a reduction of the response $(15,16)$. We detected a significant increase in the production of these chemokines when either Tyro $^{-/-}{ }^{-} \mathrm{PDL}^{+}$or PDL2 ${ }^{-}$DCs were cocultured with activated $\mathrm{CD} 4^{+} \mathrm{T}$ cells in comparison with respective WT DCs (Fig. 3D). Consistent with the requirement for OX40L in inducing $\mathrm{T}_{\mathrm{H}} 2$ responses (12), Tyro3 ${ }^{--}$PDL2 $2^{+}$DCs had higher expression of OX40L after transfer relative to WT PDL2 ${ }^{+}$DCs, whereas CD86 and CD40 levels were comparable (fig. 
S7, C and D). Finally, circulating human CD11 chi CD11b ${ }^{\text {lo }}$ DCs also expressed TYRO3. This expression was significantly increased in DCs from helminth-infected patients in comparison with healthy controls (Fig. 3E). In summary, our results indicate that the absence of TYRO3 in both PDL2 ${ }^{+}$and PDL2 ${ }^{-}$DCs leads to enhanced production of type 2 chemokines and costimulatory molecules, resulting in exacerbated type 2 immune response.

We have recently described that $\mathrm{T}$ cells, once activated, produce the TAM receptor agonist PROS1 $(2,17)$. PROS1 is also an agonist for TYRO3 $(2,17)$. However, the expression profile of PROS1 in differentiated T helper subsets was unknown. Remarkably, $\mathrm{T}_{\mathrm{H}} 2$ cells expressed PROS1 in vitro and in vivo, whereas $\mathrm{T}_{\mathrm{H}} 1$ and $\mathrm{T}_{\mathrm{H}} 17$ cells did not (fig. $\mathrm{S} 9$, A and B). These results indicated that $T$ cells differentiating toward the $\mathrm{T}_{\mathrm{H}} 2$ lineage sustain PROS1 expression, whereas expression is lost during $\mathrm{T}_{\mathrm{H}} 1$ or $\mathrm{T}_{\mathrm{H}} 17$ commitment. Gas6, the only other known agonist for TYRO3 $(17,18)$, is not expressed in murine T cells (2). Activation of T cell-receptor (TCR) signaling leads to the expression of PROS1 (2). This depends on intrinsic IL-4 production as $I L-4^{-/}$-naïve $\mathrm{CD}^{+}$T cells failed to up-regulate Pros 1 after TCR stimulation, but exogenous addition of IL-4 rescued this defect (fig. S9C). Pros1 upregulation was also STAT6-dependent (fig. S9C). Because IL-4 expression is sustained in $\mathrm{T}_{\mathrm{H}} 2$, but not in $\mathrm{T}_{\mathrm{H}} 1$ or $\mathrm{T}_{\mathrm{H}} 17$ cells, only $\mathrm{T}_{\mathrm{H}} 2$ cells maintained PROS1 expression. Only IL-4 and not IL-2, IL-5, IL-7, IL-10, IFN- $\gamma$, or transforming growth factor $\beta$ induced Pros 1 expression in naïve $\mathrm{CD} 4^{+} \mathrm{T}$ cells (fig. S9D).

To address whether PROS1 produced by T cells inhibits type 2 immune responses, we infected $\mathrm{Cd} 4 \mathrm{Cre}^{-}$Pros $f^{\text {flox/flox }}$ (control) and $\mathrm{Cd} 4 \mathrm{Cr} \mathrm{e}^{+}$Pros $\mathrm{f}^{\text {flox/flox }}$ mice with $\mathrm{N}$. brasiliensis. Activated lung CD4 ${ }^{+} \mathrm{T}$ cells of infected mice expressed PROS1 (fig. S9E). Cd4 Cre ${ }^{+}$Pros $1^{\text {flox/flox }}$ mice had fewer RBCs, increased BAL eosinophils, greater type 2 cytokine production from mLNs, and lower worm burden (Fig. 4, A to D). Furthermore, OVA-papain immunization resulted in increased leukocytes and type 2 cytokine production by antigen-specific T cells in $C d 4 \mathrm{Cr}^{+}$Pros $1^{\text {flox/flox }}$ mice (fig. S10, A to C). Last, Cd4-Cre ${ }^{+}$ Pros $f^{\text {flox/flox }}$ mice hyperresponded in a model of atopic dermatitis (fig. S10, D to F). Altogether, these findings demonstrate that IL-4 leads to sustained expression of PROS1 after $\mathrm{T}_{\mathrm{H}} 2$ differentiation. This, in turn, maintains the optimal degree of the type 2 immune response.

To explore whether this PROS1-TYRO3 axis functions in the regulation of type 2 immune responses in humans, we performed mixed-lymphocyte reactions (MLR) in the presence of an TYRO3-specific antibody or isotype control. We previously showed that human T cells express PROS1 upon activation (2). The neutralization of TYRO3 led to a significant increase in the production of IL-13 but not IFN- $\gamma$ by the T cells, which underscores a selective inhibitory role of TYRO3 in type 2, but not type 1, immunity (Fig. 4E). These results demonstrate that the TYRO3-PROS1 axis is an evolutionarily conserved negative regulator of the magnitude of the type 2 immune response.

A physiological immune response that limits the pathogen or pathogen-induced damage but avoids immunopathology has to be scalable. Such a response should have built-in sensors to monitor activation level and rheostats that vary the magnitude of the response. Here, we report the identification of a molecular mechanism that controls the intensity of type 2 
immunity. In this mechanism, a RTK in DCs and its ligand produced by $\mathrm{T}_{\mathrm{H}} 2$ cells function in concert to regulate the ensuing response. The same cytokine required for $\mathrm{T}_{\mathrm{H}} 2$ commitment after T cell priming-IL-4 (19)_induces the sustained expression of Pros 1 in T cells. This leads to the activation of the RTK TYRO3, expressed on DCs, which provides an inducible feedback mechanism at the DC-T cell interface. The overall effect of PROS1TYRO3 signaling is to limit the magnitude of the type 2 immune response.

The critical role of TYRO3 in negatively regulating $\mathrm{T}_{\mathrm{H}} 2$ responses makes this RTK an attractive pharmacological target for small molecules or biologics that activate or inhibit its function. The innate immune sensing mechanisms for the vast array of allergen and helminth products remain largely unknown. Therefore, therapeutic interventions in allergic disorders are currently limited to targeting downstream effectors of the type 2 immune response, such as histamine and leukotrienes, or to making use of broad immunosuppressive drugs, i.e., corticosteroids (20). Our discovery of a self-limiting process intrinsic to type 2 immunity provides a novel opportunity to either enhance or inhibit this response. For example, activating TYRO3 would specifically lower allergic responses by preventing adaptive immune responses that function upstream of the generation of $\operatorname{IgE}$, as well as the subsequent degranulation of eosinophils and mast cells. It could be equally attractive to extend this paradigm to parasitic infections where blocking TYRO3 may heighten type 2 response and improve expulsion of the parasites.

\title{
Supplementary Material
}

Refer to Web version on PubMed Central for supplementary material.

\section{Acknowledgments}

\begin{abstract}
We thank G. Tokmulina for cell sorting and A. Iwasaki, R. Medzhitov, J. Nussbaum, and R. Locksley for scientific discussion. The data presented in this manuscript are tabulated in the main paper and in the supplementary materials. C.V.R. is a shareholder of Kolltan Pharmaceuticals. This research was supported by grants from the American Asthma Foundation (C.V.R. and E.G.B.), NIH (R01 AI089824 to C.V.R., T32 AI007019 to P.Y.C., T32 GM007205 to D.D.K., and HL088133, HL078885, HL004464, HL104608, and HL117004 to E.G.B), the National Institute on Minority Health and Health Disparities (MD006902 to E.G.B.), Kolltan Pharmaceuticals (C.V.R.) and the Yale University Gershon-Trudeau Fellowship (P.Y.C.).
\end{abstract}

\section{REFERENCES AND NOTES}

1. Bosurgi L, et al. Proc Natl Acad Sci USA. 2013; 110:13091-13096. [PubMed: 23878224]

2. Carrera Silva EA, et al. Immunity. 2013; 39:160-170. [PubMed: 23850380]

3. Rothlin CV, Carrera-Silva EA, Bosurgi L, Ghosh S. Annu Rev Immunol. 2015; 33:355-391. [PubMed: 25594431]

4. Rothlin CV, Ghosh S, Zuniga EI, Oldstone MB, Lemke G. Cell. 2007; 131:1124-1136. [PubMed: 18083102]

5. Fujimori T, et al. Mucosal Immunol. 2015; 8:1021-1030. [PubMed: 25603826]

6. Shibata T, et al. J Immunol. 2014; 192:3569-3581. [PubMed: 24659691]

7. Hammad H, et al. J Exp Med. 2010; 207:2097-2111. [PubMed: 20819925]

8. León B, et al. Nat Immunol. 2012; 13:681-690. [PubMed: 22634865]

9. Phythian-Adams AT, et al. J Exp Med. 2010; 207:2089-2096. [PubMed: 20819926]

10. Smith KA, et al. Infect Immun. 2012; 80:3481-3489. [PubMed: 22851746]

11. Gao Y, et al. Immunity. 2013; 39:722-732. [PubMed: 24076050] 
12. Kumamoto Y, et al. Immunity. 2013; 39:733-743. [PubMed: 24076051]

13. Murakami R, et al. PLOS ONE. 2013; 8:e73270. [PubMed: 24039898]

14. Vander Lugt B, et al. Nat Immunol. 2014; 15:161-167. [PubMed: 24362890]

15. Halim TY, et al. Nat Immunol. 2016; 17:57-64. [PubMed: 26523868]

16. Perros F, Hoogsteden HC, Coyle AJ, Lambrecht BN, Hammad H. Allergy. 2009; 64:995-1002. [PubMed: 19630858]

17. Lew ED, et al. eLife. 2014; 3

18. Zagórska A, Través PG, Lew ED, Dransfield I, Lemke G. Nat Immunol. 2014; 15:920-928. [PubMed: 25194421]

19. Paul WE, Zhu J. Nat Rev Immunol. 2010; 10:225-235. [PubMed: 20336151]

20. Portelli M, Sayers I. Expert Rev Respir Med. 2012; 6:223-236. [PubMed: 22455494] 
A
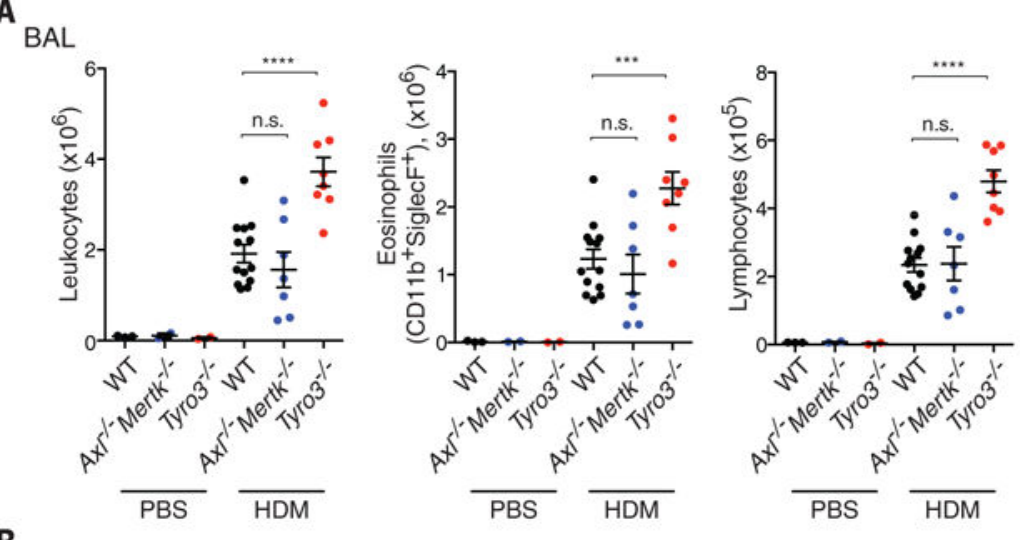

C $_{\text {Serum }}$
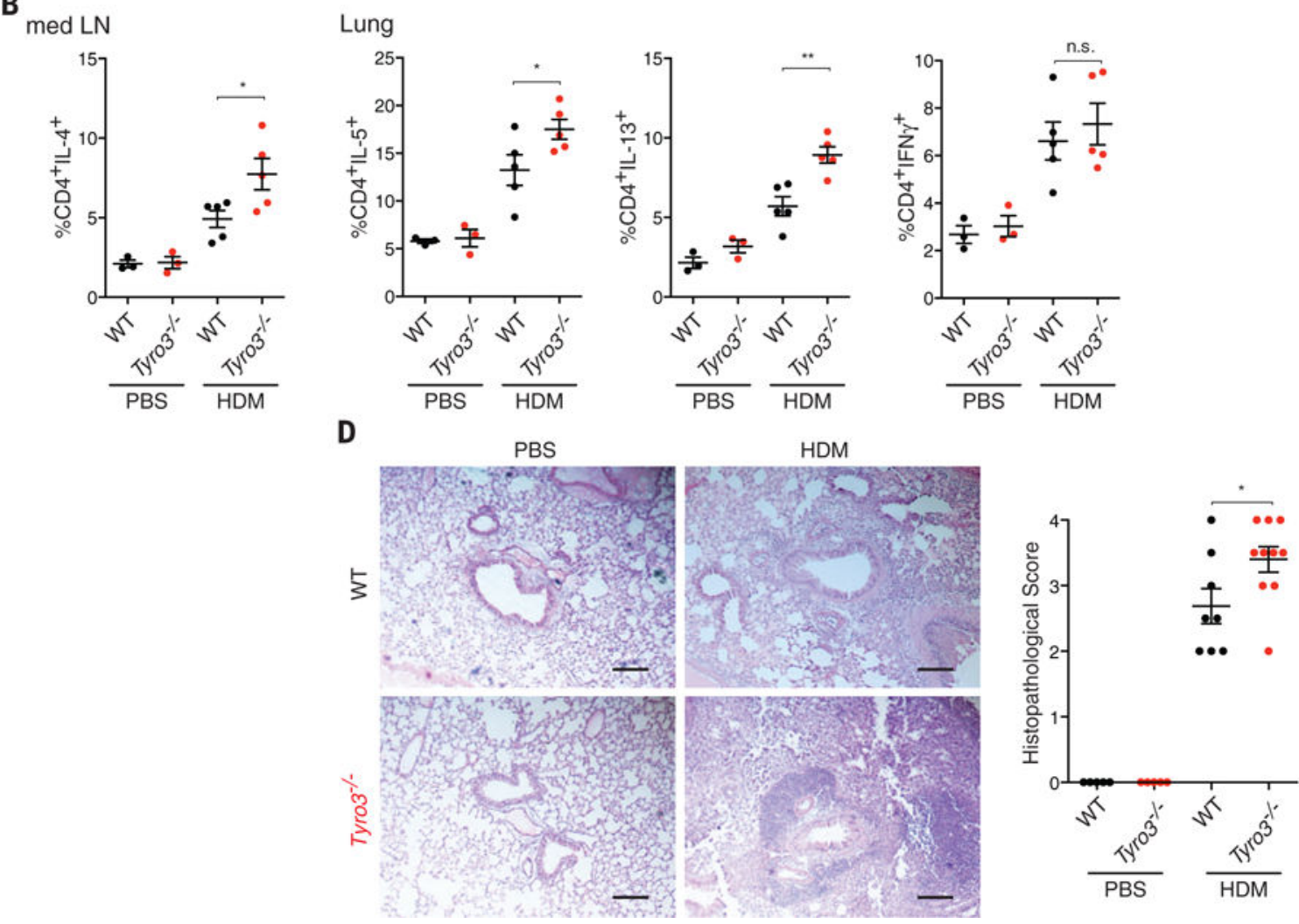

Fig. 1. Tyro $^{-/-}$mice hyperrespond to HDM-induced asthma

(A) Total numbers of infiltrating leukocytes $\left(\mathrm{CD}^{4} 5^{+}\right)$, eosinophils

$\left(\mathrm{CD}_{4}{ }^{+} \mathrm{CD} 3^{-} \mathrm{Ly}_{6 \mathrm{G}}{ }^{-} \mathrm{CD} 11 \mathrm{c}^{-} \mathrm{CD} 11 \mathrm{~b}^{+}\right.$SiglecF $\left.{ }^{+}\right)$and lymphocytes $\left(\mathrm{CD} 45^{+} \mathrm{CD}^{+}\right)$in the BAL of HDM-challenged WT, $\mathrm{Ax}^{-/} \mathrm{Mertk}^{-/-}$, and Tyro $3^{-/-}$mice. (B) Percentage of CD4 ${ }^{+} \mathrm{IL}_{-} 4^{+}$, $\mathrm{CD} 4{ }^{+} \mathrm{IL}-5^{+}, \mathrm{CD} 4^{+} \mathrm{IL}-13^{+}$, and $\mathrm{CD} 4^{+} \mathrm{IFN} \gamma^{+}$cells in medLNs and lung as indicated from phosphate-buffered saline (PBS) controls and HDM-treated mice. (C) Concentration of circulating IgE antibodies as determined by enzyme-linked immunosorbent assay (ELISA) in PBS controls and HDM-treated WT and Tyro $3^{--}$mice. (D) Representative images and independent histological scores of lung sections stained with hematoxylin and eosin from PBS controls and HDM-treated WT and Tyro $3^{-/-}$mice. Scale bar, $5 \mu \mathrm{m}$. Data are representative of two or three independent experiments. In all panels, each data point is from 
a different animal, and bars indicate means \pm SEM. Data were analyzed with analysis of variance (ANOVA) and Bonferroni multiple comparison test (A) and unpaired Student's $t$ test [(B) to (D)]. * $P 0.05 ; * * P<0.01 ; * * * P<0.001 ; * * * * P<0.0001 ;$ n.s., nonsignificant. 

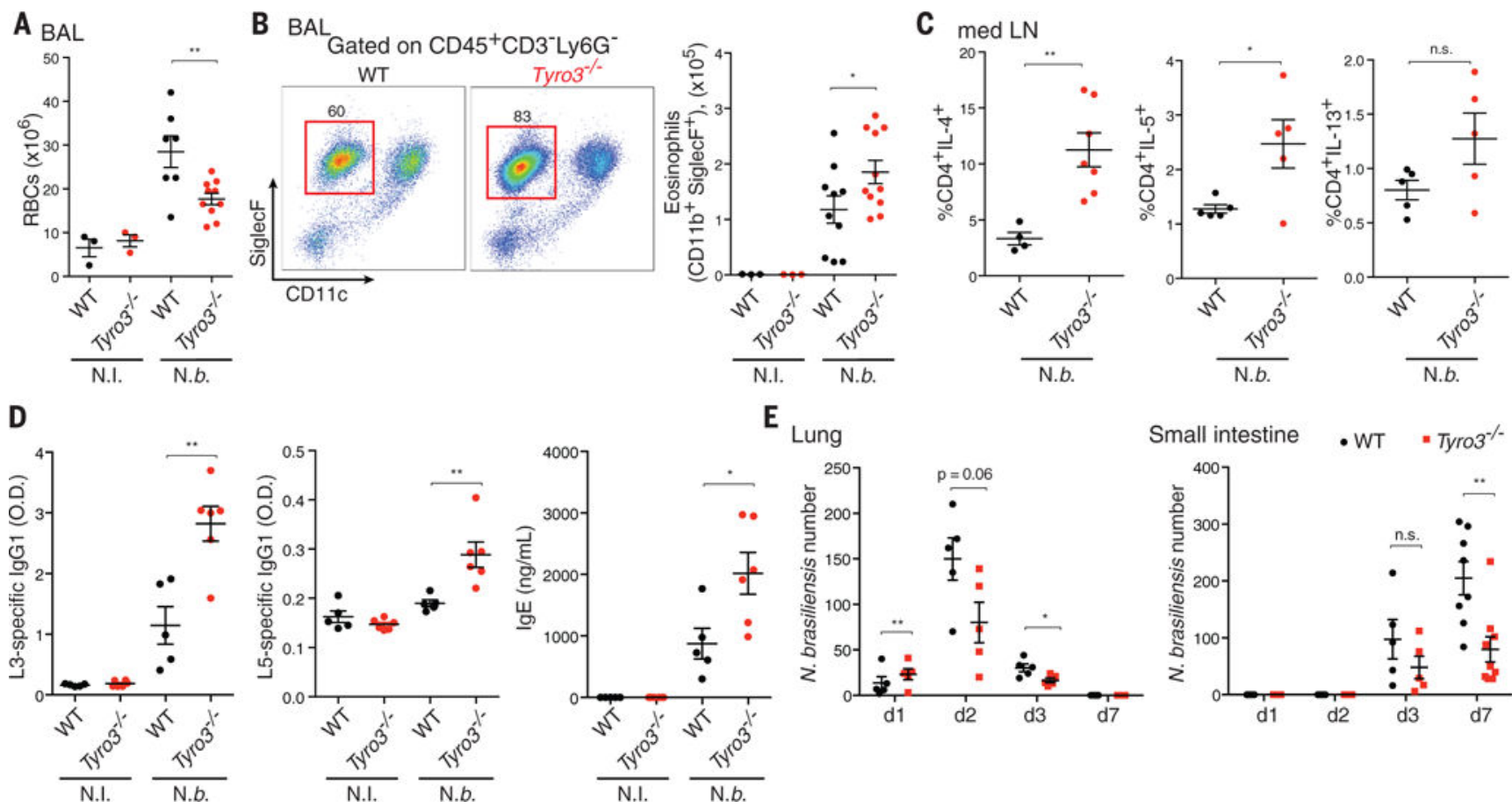

Fig. 2. Reduced damage and increased clearance in $N$. brasiliensis-infected Tyro3 $^{-/-}$mice WT and Tyro3 ${ }^{-/-}$mice were infected with 625 N. brasiliensis larvae. N.I., noninfected; N.b., $N$. brasiliensis. (A) RBC total numbers in BAL at 7 dpi. (B) Representative fluorescenceactivated cell sorting (FACS) plots of eosinophil $\left(\mathrm{CD}^{4} 5^{+} \mathrm{CD}^{-} \mathrm{Ly}^{-} \mathrm{G}^{-} \mathrm{CD} 11 \mathrm{c}^{-} \mathrm{CD}^{-11 \mathrm{~b}^{+} \text {SiglecF }}{ }^{+}\right)$percentages and total numbers of eosinophils in BAL at 7 dpi. (C) Percentage of CD4 ${ }^{+} \mathrm{IL}-4^{+}, \mathrm{CD} 4^{+} \mathrm{IL}-5^{+}$, and $\mathrm{CD} 4^{+} \mathrm{IL}-13^{+}$cells from medLNs at 2 dpi. (D) Circulating L3- and L5-specific IgG1 antibodies and total IgE antibodies as determined by ELISA. O.D., optical density. (E) Kinetics of the total parasite numbers in the lung and small intestine (S.I.) at 1 dpi, 2 dpi, 3 dpi, and 7 dpi. Data are representative of two or three independent experiments. In all graphs, each data point is from a different animal, means \pm SEM. Data were analyzed using unpaired Student's $t$ test [(A) to (E)]. $* P<0.05 ; * * P<0.01 ;$ n.s., nonsignificant. 

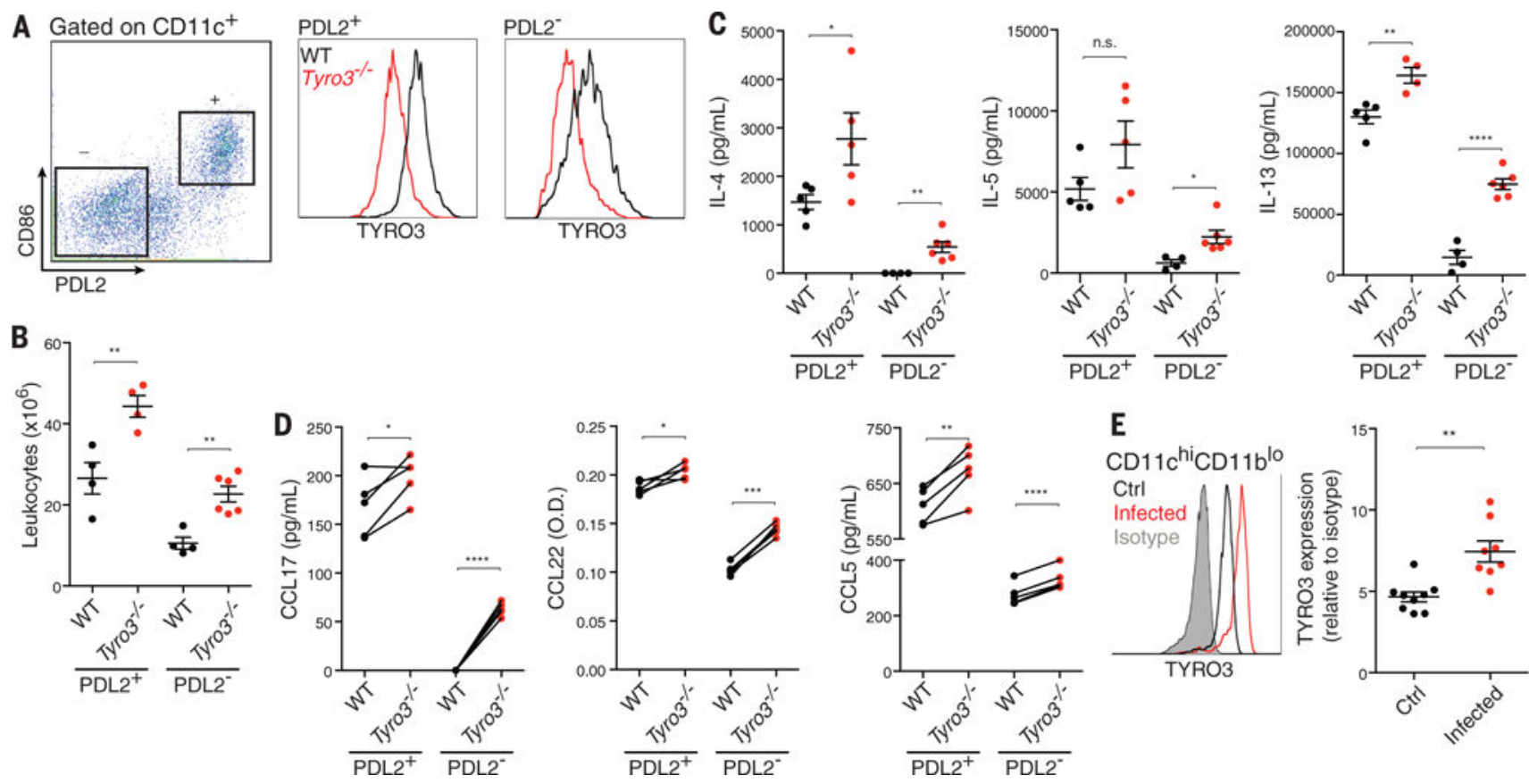

Fig. 3. TYRO3 is expressed by and functions in mouse and human DCs

(A) TYRO3 expression by FACS in sorted PDL2 ${ }^{+}$and PDL2- DCs from BM DC cultures.

(B) Total number of leukocytes from draining LNs of mice receiving WT or Tyro ${ }^{-/-}$PDL2 $^{+}$ or PDL2 ${ }^{-}$OVA-loaded DCs. (C) OVA recalls production of IL-4, IL-5, and IL-13 from $\mathrm{CD}^{+} \mathrm{T}$ cells in the draining LNs of mice receiving indicated DCs as measured by ELISA. (D) WT or Tyro3 ${ }^{-/-} \mathrm{PDL}^{+}$or PDL2 ${ }^{-}$DCs as indicated were cocultured with naïve CD4 ${ }^{+} \mathrm{T}$ cells for 24 hours in the presence of a-CD3; CCL17, CCL22, and CCL5 were measured in the supernatant by ELISA. (E) TYRO3 expression by FACS on human circulating DCs $\left(\mathrm{CD} 11 \mathrm{c}^{\mathrm{hi}} \mathrm{CD} 11 \mathrm{~b}^{\text {lo }} \mathrm{CD} 14^{\text {lo }}\right)$ from healthy controls and parasite-infected patients. Data are representative of two or three independent experiments. In all panels, each data point is from a different mouse or human. Means \pm SEM. Data were analyzed using unpaired Student's $t$ test [(B), (C), and (E)] or paired Student's $t$ test (D). ${ }^{*} P<0.05 ; * * P<0.01 ; * * * P<0.001$; $* * * * P<0.0001 ;$ n.s., nonsignificant. 
A

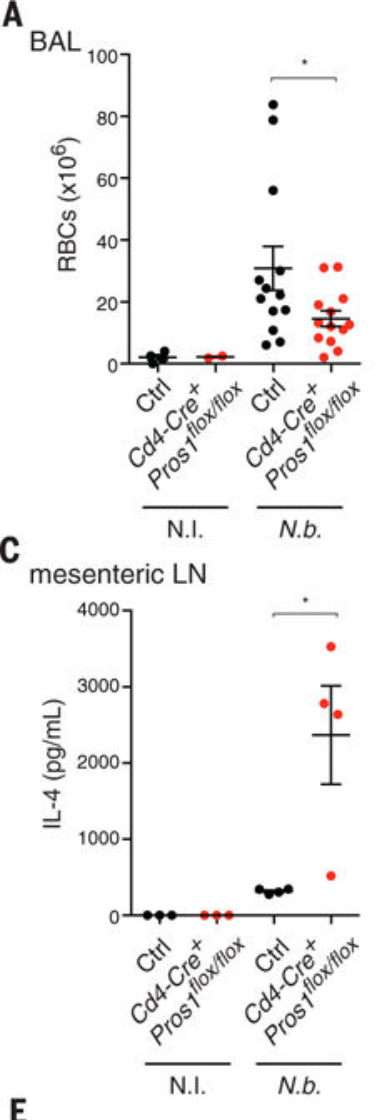

$\mathbf{E}$

Human MLR

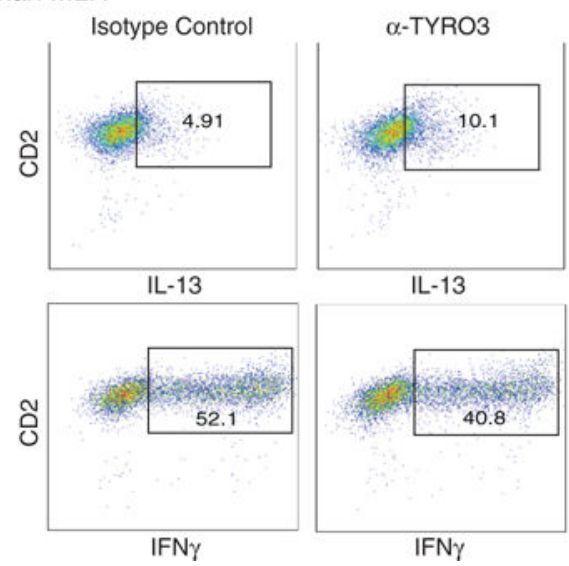

$B_{\text {BAL }}$

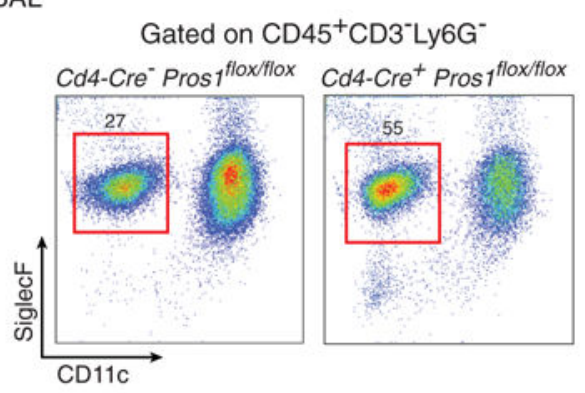

D
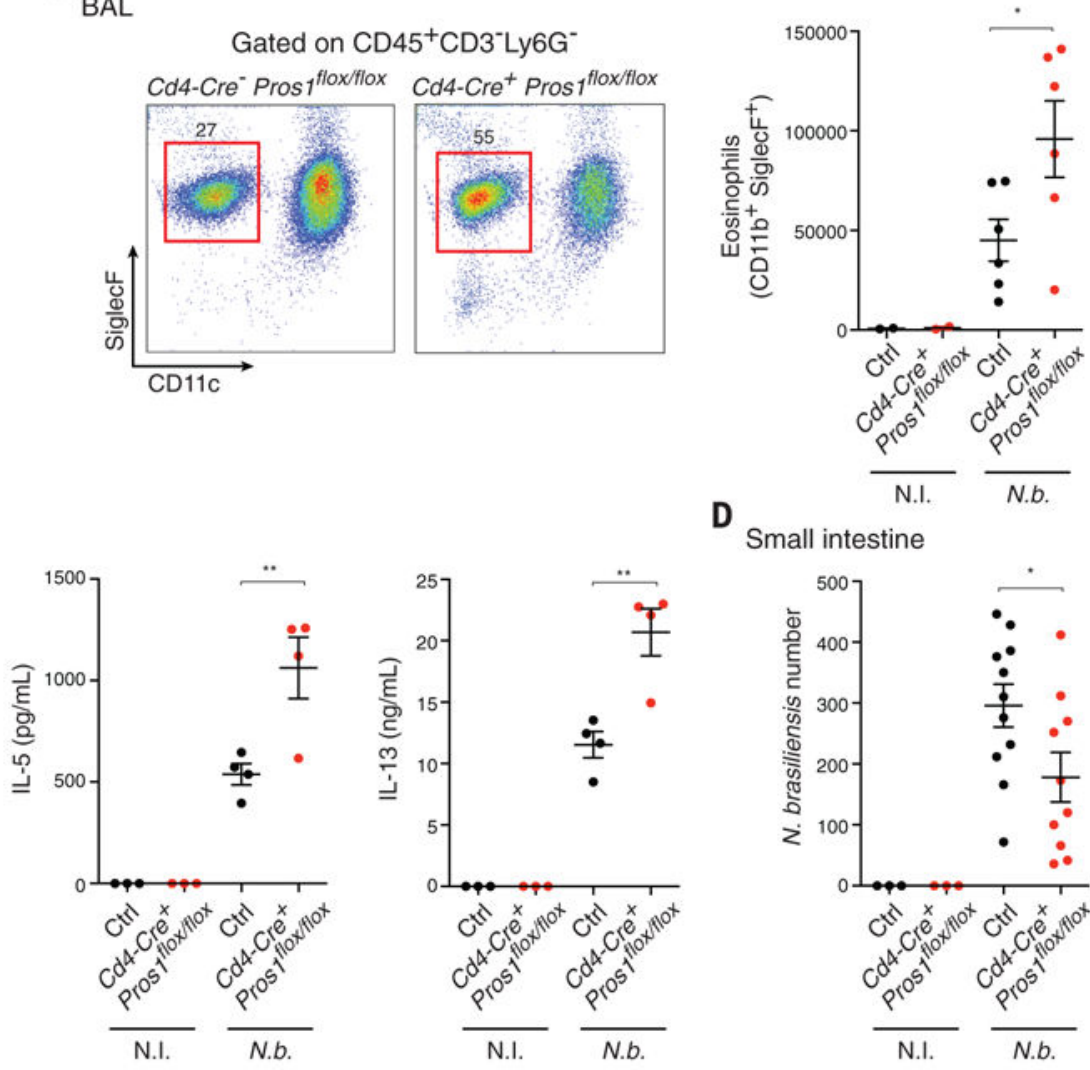

Fig. 4. T cell-derived PROS1 mediates TYRO3 regulation of type $\mathbf{2}$ immunity (A to D) Cd4-Cre-Pros $1^{\text {flox/flox }}(\mathrm{Ctrl})$ and $C d 4-C r e^{+}$Pros $1^{\text {flox/flox }}$ mice were infected with $625 \mathrm{~N}$. brasiliensis larvae, and all parameters are shown at $7 \mathrm{dpi}$. (A) RBC total numbers in BAL. (B) Representative FACS plots of eosinophil $\left(\mathrm{CD} 45^{+} \mathrm{CD}^{-} \mathrm{Ly}^{-} 6 \mathrm{G}^{-}\right.$

$\mathrm{CD} 11 \mathrm{c}^{-} \mathrm{CD} 11 \mathrm{~b}^{+}$SiglecF $\mathrm{F}^{+}$) percentages and total numbers of eosinophils in the BAL. (C) mLNs were restimulated in vitro with antibody against CD3 for 48 hours, and cytokines were measured in the supernatant by ELISA. (D) Total parasite numbers in the small intestine. (E) Representative FACS plots and independent data of the percentage of $\mathrm{CD} 2{ }^{+} \mathrm{CD} 4{ }^{+} \mathrm{IL}-13^{+}$and $\mathrm{CD} 2{ }^{+} \mathrm{CD} 4^{+} \mathrm{IFN} \gamma^{+}$cells after treating an MLR of human monocyte- 
derived DCs cocultured with human $\mathrm{CD}^{+} \mathrm{T}$ cells with either isotype antibody or antibody against TYRO3 (a-TYRO3) for 10 days. Data are representative of two or three independent experiments. In all panels, each data point is from a different mouse or human, means \pm SEM. Data were analyzed using unpaired Student's $t$ test [(A) to (D)] or paired Student's $t$ test (E). $* P<0.05, * * P<0.01, * * * P<0.001, * * * * P<0.0001$, n.s., nonsignificant. 